\title{
Highlighting advances in autophagy
}

\author{
Autophagy is a cellular degradation and recycling process with complex roles in health and disease and emerging \\ relevance to translational research. In this issue, we launch a Series of commissioned articles that will discuss \\ recent advances and outstanding questions driving this rapidly expanding and diverse field.
}

N amed from the Greek words for self (auto) and to eat (phagein), the literal meaning of autophagy is to eat oneself. Christian de Duve described autophagy in the 1960s based on the degradation of mitochondria and other cytoplasmic contents in the lysosomes of rat liver following glucagon injection. Further characterized as a process required for the survival of Saccharomyces cerevisiae in response to nutrient deprivation, autophagy is now well established as a fundamental cellular mechanism that is conserved across species. During this process, doublemembraned autophagosomes fuse with lysosomes to promote cargo degradation and release into the cytoplasm for reuse.

In the cell, autophagy is utilized in response to starvation and other stresses, and is thus critical for meeting the cell's metabolic and biosynthetic demands by replenishing macromolecular stores and-depending on the circumstancesfor promoting cell survival or death. Autophagy is also used to clear unwanted or damaged proteins and organelles, and in this capacity is important for cellular quality control and homeostasis. Years of detailed research have demonstrated that rather than being a passive degradative process, autophagy is a complex, context-dependent and tightly regulated response. The term autophagy may refer to macroautophagy, the bulk, non-selective uptake and removal of cytoplasmic contents, or to selective autophagy, which comprises the selective pathways used to identify and target specific components such as proteins, organelles or invading microorganisms, for degradation. Beyond cellular homeostasis and survival, autophagy has implications in development, immunity and inflammation, aging, and disease, including neurodegeneration and cancer. The importance of autophagy as a fundamental cellular biological process with critical roles in health and disease was underscored by the 2016 Nobel Prize in Physiology or Medicine, awarded to Yoshinori Ohsumi for his discoveries of mechanisms for autophagy.

To highlight the rapid advances in this field, we are delighted to introduce our Series on Autophagy, which launches this month with two specially commissioned Review Articles by Klionsky and co-authors
(Nat. Cell Biol. 20, 233-242; 2018), and Galluzzi and co-authors (Nat. Cell Biol. 20, 243-251; 2018)-accompanied by Research Highlights on recent autophagy studies published elsewhere. Upcoming pieces in this Series will include a Historical Perspective on autophagy by Noboru Mizushima discussing key discoveries and outstanding questions in the field. Over the subsequent months, we hope to present additional Review and Perspective Articles covering the complex molecular pathways and regulation of autophagy and its implications in cellular homeostasis, survival and pathology. The Series is hosted on a dedicated page of our website (www. nature.com/collections/ncbautophagyseries) where readers can also access an online library of related research articles published in Nature Cell Biology and the Nature journals, that will be updated as new primary and commissioned content is published.

Our Series on Autophagy begins with a Review Article by Daniel Klionsky and co-authors, which provides an in-depth view of the mechanisms that underlie the regulation of selective autophagy mechanisms, highlighting the role of ligand receptors and scaffold proteins in recognising specific cargo for degradation. The authors review how autophagy selectively degrades specific organelles and intracellular pathogens, and discuss recent progress in understanding the machinery required for selective autophagy pathways. As selective autophagy recognizes and degrades aggregateprone misfolded proteins and damaged organelles, delineating selective autophagy mechanisms has important implications for understanding associated pathologies. Recent studies have elucidated a critical role for selective degradation of mitochondria by autophagy (mitophagy) in neuronspecific and other pathologies including Huntington's, Parkinson's, and Alzheimer's diseases. Interventions targeting mitophagy may possess therapeutic potential for pathological conditions associated with mitophagy defects.

Recent years have also uncovered a complex role for autophagy in cancer, reviewed in this issue by Lorenzo Galluzzi and colleagues. The authors describe how autophagy impacts multiple aspects of oncogenesis, including cancer initiation, progression and responses to therapy. They highlight the diverse roles of autophagy in different cell types, in pre-malignant versus malignant cells and endothelial compared to other stromal cells in the tumour environment. This piece also covers progress in understanding autophagy in immune cells and implications for anticancer immunity. The emerging picture is of a multi-layered role of autophagy with anti- and protumourigenic effects in different settings, for instance depending on cancer type and disease stage. Delineating these often context- and cell-type-dependent roles and elucidating the complexity of autophagy pathways and machinery will be critical to successfully target this process in diseases such as cancer.

Studies in multicellular organisms have also revealed crucial roles for autophagy in immunity, metabolic disorders and aging. Evidence in model organisms indicates that autophagy is associated with longevity, rendering this an intriguing field of research in light of the potential implications for aging. Autophagy pathways have also been implicated in immune responses to bacterial infection and inflammatory disorders.

Although research in this field has been active for decades, much remains to be discovered. The complexity of cellular autophagic pathways continues to emerge, and the implications of this process for health and medicine keep evolving. Nature Cell Biology continues to champion this field and we look forward to publishing further key discoveries in autophagy research, with a keen interest in work that delineates the molecular mechanisms and regulation of autophagy, but also in studies that explore its implications in physiology and disease. This Series of commissioned content and the accompanying library of papers from Nature Cell Biology and the Nature journals aim to provide a snapshot into our current views of the expanding world of autophagy research. We thank our authors and referees for their contributions, and hope that you will find this Series on Autophagy informative and inspiring.

Published online: 23 February 2018 https://doi.org/10.1038/s41556-018-0061-z 